\title{
Thuộc tính bán lẻ của cửa hàng tiện lọ̣i ở các thị trường có nền kinh tế chuyển đổi: Nghiên cứu ở Việt Nam
}

\section{Retail attributes of convenience stores in transitional markets - A study in Vietnam}

\author{
Nguyễn Thanh Minh $^{1 *}$, Nguyễn Công Dũng ${ }^{1}$, Lê Anh Huyền Trâm ${ }^{1}$ \\ ${ }^{1}$ Trường Đại học Kinh Tế Thành Phố Hồ Chí Minh, Việt Nam \\ "Tác giả liên hệ, Email: minhnt@ueh.edu.vn
}

\section{THÔNG TIN}

DOI: $10.46223 / \mathrm{HCMCOUJS.}$ econ.vi.14.3.484.2019

Ngày nhận: 10/07/2019

Ngày nhận lại: 31/07/2019

Duyệt đăng: 01/08/2019

\section{Tù khóa:}

thuộc tính bán lẻ, thị trường chuyển đổi, Việt Nam, cửa hàng tiện lợi

Keywords:

convenience store, retail attributes, transitional markets, Vietnam
TÓM TẮT

Mục tiêu của nghiên cứu này nhằm khám phá các thuộc tính bán lẻ của hình thức bán lẻ cửa hàng tiện lợi ở thị trường có nền kinh tế chuyển đổi - Việt Nam. Nghiên cứu này sử dụng phương pháp nghiên cứu hỗn hợp bao gồm nghiên cứu định tính và nghiên cứu định lượng. Trong đó nghiên cứu định tính với mẫu là 20 người tiêu dùng được phỏng vấn để khám phá các thuộc tính bán lẻ của hình thức bán lẻ cửa hàng tiện lợi và nghiên cứu định lượng với mẫu 252 người tiêu dùng được khảo sát để kiểm định mô hình thuộc tính bán lẻ của cửa hàng tiện lợi. Nghiên cứu này sử dụng phương pháp phân tích yếu tố khám phá (EFA) và phương pháp phân tích nhân tố khẳng định (CFA). Kết quả nghiên cứu đã khám phá được 5 thuộc tính bán lẻ của cửa hàng tiện lợi là: tiện ích, tiện lợi giữ xe, tiện lợi lựa chọn hàng hóa, tiện lợi về vị trí và thời gian và dịch vụ khách hàng.

\section{ABSTRACT}

The purpose of this study is to explore the retail attributes of convenience stores in Vietnam, a transitional market. In this study, a research method was used to combine both qualitative and quantitative studies. In the qualitative study, 20 consumers were interviewed about the retail attributes of convenience stores and in the quantitative study, 252 consumers were surveyed to test the model of retail attributes of convenience stores. This study employed exploratory factor analysis (EFA) and confirmatory factor analysis (CFA). The findings of this study explored 5 retail attributes of convenience stores which are utilities, convenient parking, product selection convenience, location and time convenience and customer service. 


\section{1. Đặt vấn đề}

Ngành bán lẻ là ngành rất quan trọng đối với nền kinh tế của tất cả các quốc gia vì nó tạo ra hàng triệu việc làm và ảnh hưởng đáng kể đến tổng sản phẩm quốc nội của các quốc gia đó. Ở Việt Nam, doanh thu bán lẻ hàng hóa năm 2017 chiếm 58.65\% GDP và tăng 10,9\% so với năm 2016 (GSO, 2017). Ảnh hưởng đến sự phát triển của ngành bán lẻ là sự xuất hiện các hình thức bán lẻ mới, hiện đại (Tài Phan, 2018). Ở Việt Nam các hình thức bán lẻ hiện đại như siêu thị và cửa hàng tiện lợi phát triển rất nhanh (Thi Hà, 2018). Các chuỗi cửa hàng tiện lợi đang hoạt động là Circle K, Ministop, Zakka Mart, Shop \& Go, B's mart, FamilyMart, VinMart+...số lượng cửa hàng tiện lợi tăng nhanh qua các năm. Từ năm 2014 đến năm 2018 số lượng cửa hàng tăng 3,5 lần từ 326 tới 1.144 cửa hàng ở Thành phố Hồ Chí Minh (Thi Hà, 2018). Tuy nhiên lại có nhiều hệ thống cửa hàng tiện lợi thất bại, đóng cửa như G7, Vissan và tốc độ mở rộng, phát triển chậm không như mong muốn của FamilyMart, 7-Eleven, Ministop (Hiếu Công, 2018; Thi Hà, 2018). Điều này đặt ra câu hỏi lớn về mô hình kinh doanh thành công của cửa hàng tiện lợi. Trong các yếu tố ảnh hưởng đến sự thành công của cửa hàng tiện lợi thì việc hiểu thói quen và hành vi tiêu dùng là rất quan trọng. Sự thay đổi thói quen và hành vi mua sắm của người tiêu dùng dẫn tới việc đặt ra câu hỏi cho các nhà nghiên cứu về các thuộc tính bán lẻ quan trọng của cửa hàng tiện lợi đối với người tiêu dùng, đặc biệt sự thay đổi này xảy ra ở các thị trường có nền kinh tế chuyển đổi, mới nổi - là các thị trường có những đặc điểm khác biệt và quan trọng đối với các nhà nghiên cứu và bán lẻ (Batra, 1997; T. M. T. Nguyen \& T. D. Nguyen, 2003).

Trong các nghiên cứu về các thuộc tính bán lẻ của các hình thức bán lẻ hiện đại ở các thị trường mới nổi, có nền kinh tế chuyển đổi như Việt Nam, hầu hết các nghiên cứu chỉ tập trung vào hình thức bán lẻ là siêu thị (T. T. M. Nguyen, Nigel, \& T. D. Nguyen, 2007). Đối với hình thức bán lẻ là cửa hàng tiện lợi thì chưa có nhiều nghiên cứu về các thuộc tính bán lẻ. Do đó, nghiên cứu này sẽ tập trung nghiên cứu thuộc tính bán lẻ của cửa hàng tiện lợi và thực hiện ở thị trường mới nổi là Việt Nam. Nghiên cứu này có các mục tiêu sau đây:

Mục tiêu thứ nhất là khám phá các thuộc tính bán lẻ của hình thức bán lẻ là cửa hàng tiện lợi tại thị trường mới nổi có nền kinh tế chuyển đổi Việt Nam.

Mục tiêu thứ hai là so sánh sự khác biệt của các thuộc tính bán lẻ của cửa hàng tiện lợi ở thị trường có nền kinh tế chuyển đổi Việt Nam với các thuộc tính bán lẻ của cửa hàng tiện lợi đã được nghiên cứu ở các thị trường các nước phát triển.

\section{Nghiên cứu lý thuyết}

\section{1. Định nghĩa thuộc tính bán lẻ và cửa hàng tiện lọi}

\subsection{1. Định nghĩa cửa hàng tiện lọi}

Theo Hiệp hội cửa hàng tiện lợi Mỹ (NACS, 2016), cửa hàng tiện lợi được định nghĩa là một hoạt động kinh doanh bán lẻ chủ yếu nhấn mạnh vào vị trí tiện lợi công cộng để khách hàng có thể mua nhanh chóng các sản phẩm tiêu dùng (chủ yếu là thức ăn hoặc mua xăng dầu). Trong nghiên cứu này, cửa hàng tiện lợi được định nghĩa là những cửa hàng bán lẻ hiện đại, có vị trí tiện lợi, giờ hoạt động lâu, bán các mặt hàng thiết yếu, tiêu dùng nhanh. Ví dụ như là cửa hàng tiện lợi Shop \& Go, Ministop, Family Mart, 7 Eleven... 


\subsubsection{Sự phát triển của cửa hàng tiện lợi trên thế giới}

Theo Garves (2017), cửa hàng tiện lợi đầu tiên xuất hiện ở Mỹ năm 1927 bởi Jefferson Green và các hệ thống cửa hàng tiện lợi phát triển rất nhanh (Bianchi, 2009). Ở Đan Mạch và Thuỵ Điển việc phát triển của hệ thống cửa hàng lợi dựa trên sự liên kết với các trạm xăng dầu và ở Anh và Pháp thì sự phát triển này lại kết nối với ngành công nghiệp thực phẩm (David \& Kirby, 1986; Zairis \& Evangelos, 2014). Ở Nhật Bản, hệ thống cửa hàng tiện lợi phát triển với một tốc độ nhanh chóng với việc sử dụng các công nghệ tiên tiến (Ogawa, 2002; Zairis \& Evangelos, 2014). Hai hệ thống cửa hàng tiện lợi nổi tiếng của Nhật Bản là Family Mart và 7 Eleven Japan. (Thanh Ngọc, 2016). Ở Đài Loan, hệ thống cửa hàng tiện lợi phát triển từ năm 1977 và mật độ cửa hàng ở Đài Loan hiện nay được xem là cao nhất thế giới (Antonios \& Prontzas, 2014; Cheng, Blankson, Sutikno, \& Wang, 2009). Từ các nghiên cứu trên, có thể nhận thấy cửa hàng tiện lợi là một hình thức bán lẻ quan trọng.

\subsubsection{Sự phát triển của cửa hàng tiện lợi ở Việt Nam}

Từ khi mở cửa cho thị trường bán lẻ vào năm 2009 và các rào cản được giảm bớt và doanh nghiệp nước ngoài được mở các cửa hàng có diện tích dưới $500 \mathrm{~m} 2$, Việt Nam đã tạo điều kiện thuận lợi cho việc phát triển các hệ thống cửa hàng tiện lợi (Hiếu Công, 2018). Theo Nielsen Việt Nam thì tăng trưởng của hệ thống cửa hàng tiện lợi ở Việt Nam đạt mức cao, năm 2014 tăng 34,4\% so với 2013, năm 2015 tăng $75 \%$ so với 2014, vượt xa mức tăng trưởng $6 \%$ của siêu thị và đại siêu thị và trong tương lai số lượng cửa hàng tiện lợi sẽ có thể tăng gấp 3 lần (A. Nguyen, 2015; Đông Nghi, 2015). Hiện tại ở Việt Nam có nhiều chuỗi cửa hàng tiện lợi như Shop \& Go (Singapore), Circle K (Mỹ), FamilyMart (Nhật), B's Mart (Thái Lan), MiniStop (Nhật Bản), Zakka Mart (Việt Nam), VinMart+ (Việt Nam) ... (Đông Nghi, 2015; Thế Trần, 2016).

Mặc dù, sự phát triển của thị trường bán lẻ Việt Nam là rõ ràng nhưng nhiều hệ thống cửa hàng tiện lợi vẫn gặp nhiều khó khăn trong việc thâm nhập thị trường Việt Nam và gặp thua lỗ và thất bại. Chuỗi cửa hàng tiện lợi đầu tiên ở Việt Nam là G7 Mart của Trung Nguyên được thành lập năm 2006 nhưng hoạt động kinh doanh của G7 không thành công do người tiêu dùng Việt Nam khi đó chỉ quan tâm tới giá bán hơn là sự tiện lợi như thời gian mua sắm, sự phục vụ (A. Nguyen, 2015). Sau đó Trung Nguyên áp dụng mô hình nhượng quyền với hệ thống Ministop từ Nhật Bản nhưng cũng không thành công và phải kết thúc mối quan hệ hợp tác. Vissan, một doanh nghiệp Việt Nam khác cũng phải đóng cửa khoảng 60 cửa hàng tiện lợi trong 100 cửa hàng của mình (Hiếu Công, 2018). Các hệ thống cửa hàng tiện lợi nước ngoài như Family Mart cũng đang tạm ngừng đầu tư và đang gặp thua lỗ. 7-Eleven mới chỉ mở được 18 cửa hàng khi mục tiêu hằng năm là 100 cửa hàng (Hiếu Công, 2018). Các lý do được đưa ra là do hành vi, thói quen mua sắm của người tiêu dùng Việt Nam chưa quen với hình thức bán lẻ mới này, hình thức này có thể chưa đáp ứng được nhu cầu mua sắm của họ, chi phí mặt bằng cao và phải cạnh tranh với các cửa hàng tạp hóa truyền thống và các siêu thị (Hiếu Công, 2018; Thi Hà, 2018). Trong các lý do này thì lý do về hành vi mua sắm của người tiêu dùng được coi là một trong những lý do quan trọng nhất. Nếu các hệ thống cửa hàng tiện lợi có thể nhận biết được các thuộc tính bán lẻ quan trọng thì sẽ có thể thu hút được sự lựa chọn mua sắm của người tiêu dùng thì có thể dễ dàng hơn trong việc thâm nhập vào thị trường. Do đó, việc nghiên cứu 
các thuộc tính bán lẻ của cửa hàng tiện lợi trong nghiên cứu này có thể giúp các nhà bán lẻ hiểu biết hơn về nhu cầu, sở thích khi đi mua sắm của người tiêu dùng.

\subsubsection{Thuộc tính bán lẻ và thuộc tính bán lẻ "tiện lợi"}

Thuộc tính bán lẻ được định nghĩa là sự tổng hợp tất cả các thuộc tính của một cửa hàng được cảm nhận bởi người mua sắm qua trải nghiệm mua sắm của họ ở cửa hàng đó (Omar, trích dẫn bởi T. T. M. Nguyen et al., 2007). Mặc dù nghiên cứu về thuộc tính bán lẻ được thực hiện rộng rãi ở nhiều nước trên thế giới nhưng các nghiên cứu này chỉ được thực hiện nghiên cứu chủ yếu cho các hình thức bán lẻ như siêu thị, cửa hàng chiết khấu, cửa hàng quần áo, trung tâm thương mại, cửa hàng kho, mua sắm trên internet, cửa hàng bách hóa, cửa hàng chuyên doanh, cửa hàng tạp hóa (Jason \& Marguerite, 2006; Jin \& Kim, 2003; Koo, 2003; Zielke, 2010)...Ngoài ra, chưa có nhiều các nghiên cứu về thuộc tính bán lẻ của cửa hàng tiện lợi. Mặc dù có thể cho rằng người tiêu dùng chọn mua sắm ở các cửa hàng tiện lợi vì sự tiện lợi. Tuy nhiên thuộc tính bán lẻ "tiện lợi” vẫn có thể được hiểu rất khác nhau và có nhiều tranh luận về ý nghĩa của thuộc tính "tiện lợi” trong bán lẻ do không có định nghĩa rõ ràng về sự tiện lợi (Seiders, Berry, \& Gresham, 2000). Ví dụ: nếu cửa hàng có nhiều lối ra vào hay cửa hàng có kệ để hàng có độ cao phù hợp có thể được là mang lại tiện lợi cho khách hàng (Reimers, 2014). Theo Reimers (2014), có 25 thuộc tính bán lẻ liên quan đến sự tiện lợi. Do vậy thuộc tính tiện lợi có thể được hiểu khác nhau và có ý nghĩa khác nhau tùy theo văn hóa của thị trường.

\subsection{Các nghiên cứu về thuộc tính bán lẻ của cửa hàng tiện lội đã đự̣c thục hiện}

Ở Anh, Wood và Browne (2007) đã nghiên cứu vai trò thuộc tính vị trí cửa hàng tiện lợi (location) để lập kế hoạch bán lẻ. Ở Pháp, Gahinet và Cliquet (2018) chỉ nghiên cứu 2 thuộc tính của cửa hàng tiện lợi đó là sự gần gũi (proximity) và sự tiện lợi thời gian ảnh hưởng tới lòng trung thành của người tiêu dùng. Tuy nhiên, Wood và Browne (2007) và Gahinet và Cliquet (2018) đã không nghiên cứu đầy đủ các thuộc tính bán lẻ của cửa hàng tiện lợi.

Ở Nhật, Rapp và Islam (2006) nghiên cứu thuộc tính dịch vụ của cửa hàng tiện lợi. Các cửa hàng tiện lợi ở Nhật đã cung cấp các dịch vụ của các ngân hàng bán lẻ. Các cửa hàng này hoạt động như các ngân hàng nhỏ cho vay, thanh toán các hóa đơn dịch vụ, thanh toán online, chuyển tiền và thẻ tín dụng. Ngoài ra, ở Nhật, Hsu và Huang (2006) còn nghiên cứu thuộc tính dịch vụ với hình thức phân phối điện tử qua các website. Các cửa hàng tiện lợi liên kết với các công ty khác cung cấp nhiều loại sản phẩm và dịch vụ như du lịch, nhạc, hình ảnh, thanh toán hóa đơn, sách, cho thuê ô tô và điện thoại.

Ở Đài Loan, Hsu và Huang (2006) đề nghị các cửa hàng tiện lợi còn đóng vai trò làm đại lý và logistic cho các công ty khác. Cheng và cộng sự (2009) đề nghị một thuộc tính của cửa hàng tiện lợi ở Đài Loan là cung cấp dịch vụ ăn tối cho khách hàng. Khác với các loại thức ăn nhanh thường thấy trong các cửa hàng tiện lợi, dịch vụ ăn tối này được cung cấp trong một khu vực riêng của cửa hàng tiện lợi và khu vực này được thiết kế như một nhà hàng thức ăn nhanh hiện đại với nhiều bàn ghế và nhiều lựa chọn hơn và vệ sinh hơn. Tuy nhiên, các thuộc tính đầy đủ của cửa hàng tiện lợi cũng chưa được nghiên cứu trong các nghiên cứu ở Nhật và Đài Loan. 
Ở khu vực châu Mỹ Latinh, Bianchi (2009) đã nghiên cứu về các thuộc tính bán lẻ của cửa hàng tiện lợi với 400 người tiêu dùng là các sinh viên đại học và sau đại học ở Chi Lê và đề nghị các thuộc tính quan trọng của cửa hàng tiện lợi là cửa hàng có thể được tiếp cận nhanh chóng (Quick access to the store), nhiều hình thức thanh toán và chỗ đậu xe, gần nhà, nhân viên phục vụ, giờ hoạt động, sạch sẽ và gọn gàng, hàng hóa có thể đổi trả lại, môi trường mua sắm an toàn, chủng loại hàng hóa, giá thấp, gần nơi làm việc, cách bố trí cửa hàng, các hình thức chiết khấu và bàn ghế cho khách hàng. Tuy nhiên nghiên cứu này, Bianchi (2009) chỉ dùng phương pháp thống kê đơn giản như mô tả, Cross-tab, so sánh trung bình. Ngoài ra, mẫu nghiên cứu là các sinh viên đại học và sau đại học nên mẫu chưa thật sự đại diện người tiêu dùng.

Ở Hy Lạp, Zairis và Evangelos (2014) đã nghiên cứu 360 người mua sắm tại các cửa hàng tiện lợi để xác định các thuộc tính bán lẻ quan trọng như vị trí thuận tiện (proximity), dịch vụ có chất lượng cao, giá rẻ và hàng hóa phong phú. Tuy nhiên, Zairis và Evangelos (2014) chỉ dùng thống kê mô tả để tìm hiểu mức độ ưa chuộng của người tiêu dùng về các hệ thống cửa hàng tiện lợi.

Từ các nghiên cứu đã được thực hiện và được trình bày ở trên, có thể thấy rất hạn chế các nghiên cứu chi tiết, đầy đủ về các thuộc tính bán lẻ của cửa hàng tiện lợi. Các nghiên cứu về thuộc tính bán lẻ của cửa hàng tiện lợi đã được thực hiện thì có số lượng ít và hầu hết các nghiên cứu này được thực hiện ở các nước phát triển (Cheng et al., 2009; Hsu và Huang, 2006; Rapp \& Islam, 2006; Sutton \& Rafaeli, 1988; Wood \& Browne, 2007; Zairis \& Evangelos, 2014), ngoại trừ một nghiên cứu của Bianchi (2009) được thực hiện Chi Lê, một thị trường mới nổi ở Mỹ La Tinh. Ngoài ra chưa có nghiên cứu được thực hiện ở một nước đang phát triển và có nền kinh tế chuyển đổi. Điều này dẫn tới sự cần thiết của việc nghiên cứu ở các thị trường mới nổi và có nền kinh tế chuyển đổi để có thể cung cấp sự hiểu biết đầy đủ hơn về hành vi của người tiêu dùng vì người tiêu dùng sẽ có những quan tâm các thuộc tính bán lẻ khác nhau do sự khác biệt về văn hoá và sự phát triển kinh tế (Bianchi, 2009; Kim \& Kang, 1995). Vì Việt Nam là một thị trường mới nổi và có nền kinh tế chuyển đổi và là một thị trường thu hút nhiều sự chú ý của nhiều nhà nghiên cứu (T. M. T. Nguyen \& T. D. Nguyen, 2003) nên nghiên cứu này được thực hiện ở Việt Nam. Các thuộc tính như dịch vụ du lịch, nhạc, hình ảnh, thanh toán hóa đơn, sách, cho thuê ô tô và điện thoại, dịch vụ ngân hàng dù được ưa chuộng ở các nước phát triển như Nhật, Đài Loan... nhưng có thể chưa được người tiêu dùng ở thị trường chuyển đổi như Việt Nam quan tâm. Do chưa có nghiên cứu đầy đủ và hệ thống về thuộc tính bán lẻ của cửa hàng tiện lợi nói chung và cũng như ở thị trường chuyển đổi như Việt Nam, nghiên cứu này sẽ nghiên cứu thuộc tính bán lẻ cửa hàng tiện lợi.

\subsection{Giả thuyết nghiên cúu và Mô hình nghiên cúu}

Từ kết quả nghiên cứu định tính đã được thực hiện phỏng vấn 20 người tiêu dùng trong nghiên cứu này (sẽ được trình bày trong phần phương pháp nghiên cứu) đã xác định là Tiện lợi về vị trí và thời gian là thuộc tính quan trọng của cửa hàng tiện lợi. Cửa hàng tiện lợi có vị trí thuận tiện cho việc mua sắm: vì vị trí cửa hàng tiện lợi thường ở các ngã tư, ngã ba, trên đường phố và gần trường học, các cao ốc văn phòng, chung cư, khu vui chơi giải trí hoặc trung tâm thành phố tập trung đông người. Người mua sắm khi đi bộ hoặc xe máy, ô tô có thể ghé vào mua sắm nhanh món hàng họ muốn. Các nghiên cứu trước đây cũng đề nghị sự tiện lợi về vị trí 
và thời gian là thuộc tính quan trọng của cửa hàng tiện lợi (Gahinet \& Cliquet, 2018; Zairis \& Evangelos, 2014). Do vậy, trong thị trường Việt Nam, nghiên cứu này đề nghị giả thuyết:

\section{H1: Tiện lợi về vị trí và thời gian là thuộc tính quan trọng của cưa hàng tiện lợi}

Cửa hàng tiện lợi cung cấp các tiện ích tiện lợi cho mua sắm: khi mua sắm người mua sắm có thể vào đây để nghỉ chân vì có sẵn bàn ghế, điều hòa. Các cửa hàng tiện lợi còn trang bị nhiều bàn ghế để người mua sắm có thể ngồi ăn uống các thức ăn được mua ở cửa hàng tiện lợi và người mua sắm có thể sử dụng bàn ghế làm việc riêng, gặp người thân quen cùng với các dịch vụ wifi miễn phí và sạc điện thoại. Hsu và Huang (2006) cũng đề nghị các tiện ích tại nơi mua sắm như có khu vực ăn uống, bàn ghế... là thuộc tính bán lẻ quan trọng của cửa hàng tiện lợi trong nghiên cứu ở Đài Loan. Do đó, nghiên cứu này đề nghị giả thuyết:

\section{H2: Tiện ích là thuộc tính quan trọng của cửa hàng tiện lọii}

Thuộc tính bãi giữ xe là thuộc tính quan trọng của cửa hàng tiện lợi ở Mỹ nhưng ở Nhật thì không quan trọng (Akinobu, 1998; Zairis \& Evangelos, 2014). Tuy nhiên, ở Việt Nam, người dân chủ yếu sử phương tiện đi lại cá nhân như xe máy, xe đạp...Khi mua sắm ở cửa hàng tiện lợi, người mua sắm có thể dễ dàng đậu xe và gửi xe không tốn phí, khác với siêu thị hay chợ truyền thống, người mua sắm thường phải gửi xe mất thời gian tìm nơi cung cấp dịch vụ gửi xe và tốn phí gửi xe. Do vậy, trong thị trường Việt Nam, nghiên cứu này đề nghị giả thuyết:

\section{H3: Tiện lợi giũ xe là thuộc tính quan trọng của cửa hàng tiện lợi}

Hàng hóa ở cửa hàng tiện lợi không quá nhiều và phong phú như siêu thị nên người mua dễ dàng tìm kiếm và lựa chọn mua. Ngoài ra, giá bán tại cửa hàng tiện lợi là cố định; người mua không phải trả giá, thương lượng trong chợ truyền thống nên người mua có thể dễ dàng so sánh giá và lựa chọn hàng hóa dễ dàng mà không bị làm phiền bởi người bán như trong chợ truyền thống. Jason và Marguerite (2006) cũng đề nghị thuộc tính lựa chọn hàng hóa là thuộc tính bán lẻ quan trọng ảnh hưởng người tiêu dùng khi lựa chọn nơi mua sắm. Do vậy, trong thị trường Việt Nam, nghiên cứu này đề nghị giả thuyết:

\section{H4: Tiện lợi lựa chọn hàng hóa là thuộc tính quan trọng của của hàng tiện lợi}

Cửa hàng tiện lợi có nhân viên được đào tạo, huấn luyện chuyên nghiệp nên phục vụ tốt, đúng quy trình và làm hài lòng khách hàng. Họ thường chào hỏi khách hàng khi khách hàng vào mua sắm hơn như khi họ mua sắm ở siêu thị và chợ truyền thống. Ở Hy Lạp, nhân viên thân thiện/dịch vụ khách hàng được xem là thuộc tính quan trọng của cửa hàng tiện lợi (Zairis \& Evangelos, 2014). Do vậy, trong thị trường Việt Nam, nghiên cứu này đề nghị giả thuyết:

H5: Nhân viên thân thiện/Dịch vụ khách hàng là thuộc tính quan trọng của cưa hàng tiện lợi

\section{Phương pháp nghiên cứu}

Trong nghiên cứu này, phương pháp nghiên cứu hỗn hợp được sử dụng bao gồm phương pháp nghiên cứu định tính và phương pháp nghiên cứu định lượng. Nghiên cứu định tính có mục đích nhằm khám phá, điều chỉnh và bổ sung các thuộc tính bán lẻ của cửa hàng tiện lợi. Trong phương pháp định tính, số lượng người tiêu dùng được phỏng vấn là 20 . Những người tiêu dùng này đã từng mua sắm tại các cửa hàng tiện lợi với các độ tuổi khác nhau và giới tính, 
thu nhập khác nhau với phương pháp chọn mẫu thuận tiện. Số lượng người tiêu dùng dừng ở số 20 được phỏng vấn do thông tin thu thập được trong nghiên cứu định tính không có khám phá mới (bão hòa). Người tiêu dùng được tiếp cận ở các công viên, phố đi bộ, trung tâm Thành phố Hồ Chí Minh và được giới thiệu về nội dung nghiên cứu và sau khi được người tiêu dùng đồng ý tham gia thì người tiêu dùng được phỏng vấn sâu khoảng 30 đến 45 phút với các thuộc tính bán lẻ của cửa hàng tiện lợi khi họ đi mua sắm ở các cửa hàng này như Shop \& Go, Circle K, FamilyMart, B’s Mart, MiniStop, Zakka Mart, VinMart+... Các kết quả của phỏng vấn sẽ được ghi chép chi tiết để phân tích sau đó nhằm tìm ra các thuộc tính bán lẻ của cửa hàng tiện lợi. Từ các kết quả này, bảng câu hỏi chi tiết sẽ được thiết kế cho nghiên cứu định lượng. Nghiên cứu sơ bộ bằng phương pháp định tính được thực hiện vào tháng $1 / 2018$. Nghiên cứu định lượng tiếp theo được thực hiện bằng phương pháp phỏng vấn trực tiếp người tiêu dùng với phương pháp chọn mẫu thuận tiện. Tương tự nghiên cứu định tính, người tiêu dùng được tiếp cận ở các công viên, phố đi bộ, trung tâm Thành phố Hồ Chí Minh và được giới thiệu về nội dung nghiên cứu và sau khi được người tiêu dùng đồng ý tham gia nghiên cứu thì người tiêu dùng được mời trả lời bảng câu hỏi khảo sát. Trong bảng câu hỏi khảo sát, các thuộc tính bán lẻ được đo lường bằng thang đo quãng 5 điểm theo tầm quan trọng trong đó 1 : không quan trọng và 5 : rất quan trọng. Các thuộc tính bán lẻ được thu thập từ lý thuyết và nghiên cứu định tính được trình bày trong Bảng 1 . Kích thước mẫu cho nghiên cứu chính thức định lượng này là 252 người tiêu dùng. Độ tuổi của người được phỏng vấn là từ 18 trở lên. Những người được chọn là những người tiêu dùng đã từng mua sắm tại các cửa hàng tiện lợi như Shop \& $\mathrm{Go}$, Circle $\mathrm{K}$, FamilyMart, B's Mart, MiniStop, Zakka Mart, VinMart+... Mẫu được lựa chọn ở Thành phố Hồ Chí Minh vì đây là thành phố lớn ở Việt Nam và có nhiều hệ thống cửa hàng tiện lợi. Dữ liệu được phân tích bằng phần mềm SPSS. Phương pháp phân tích nhân tố khẳng định CFA được sử dụng trong nghiên cứu này để kiểm định mô hình nghiên cứu do phương pháp này có nhiều ưu điểm hơn các phương pháp khác như phương pháp phân tích nhân tố EFA, hay hệ số tương quan...(T. M. T. Nguyen \& T. D. Nguyen, 2003). Trong các nghiên cứu trước đây về thuộc tính bán lẻ của cửa hàng tiện lợi đã được trình bày ở phần trên, các nghiên cứu này chỉ sử dụng một số phương pháp thống kê đơn giản như mô tả, so sánh trung bình như Bianchi (2009), Zairis và Evangelos (2014)...

\section{Kết quả và thảo luận}

\subsection{Kết quả nghiên cúu so bộ (định tính)}

Nghiên cứu sơ bộ định tính phỏng vấn 20 người tiêu dùng thường mua sắm ở cửa hàng tiện lợi ở Thành phố Hồ Chí Minh với 10 người giới tính Nam và 10 người giới tính Nữ, độ tuổi từ 18 đến 50 và thu nhập khác nhau từ 2 triệu tới trên 10 triệu một tháng. Từ việc phỏng vấn sâu người tiêu dùng (in-depth interview) ở Thành phố Hồ Chí Minh, nghiên cứu định tính đã xác nhận và khám phá ra 23 thuộc tính bán lẻ của cửa hàng tiện lợi.

\section{Bảng 1}

Kết quả nghiên cứu sơ bộ (định tính)

\begin{tabular}{|c|l|c|}
\hline STT & \multicolumn{1}{|c|}{ Thuộc tính bán lẻ cửa hàng tiện lọi } & Nguồn \\
\hline 1 & Nơi làm việc, học bài & Nghiên cứu định tính \\
\hline
\end{tabular}




\begin{tabular}{|c|c|c|}
\hline STT & Thuộc tính bán lẻ cửa hàng tiện lọ̣i & Nguồn \\
\hline 2 & Nơi có thể nói chuyện với người quen & Nghiên cứu định tính \\
\hline 3 & Nơi nghỉ chân thư giãn & Nghiên cứu định tính \\
\hline 4 & Nơi để gặp gỡ mọi người & $\begin{array}{l}\text { M. T. Nguyen (2014) và Nghiên cứu định } \\
\text { tính }\end{array}$ \\
\hline 5 & Nơi để giết thời gian & Ugur (2003) và Nghiên cứu định tính \\
\hline 6 & Có wifi miễn phí, sạc điện thoại & Nghiên cứu định tính \\
\hline 7 & Có khu vực ăn uống & $\begin{array}{l}\text { Jason và Marguerite (2006) và Nghiên } \\
\text { cứu định tính }\end{array}$ \\
\hline 8 & Có thể tự phục vụ & Nghiên cứu định tính \\
\hline 9 & $\begin{array}{l}\text { Có dịch vụ thanh toán, điện nước, internet, } \\
\text { ATM }\end{array}$ & Nghiên cứu định tính \\
\hline 10 & Không tốn tiền gửi xe & Nghiên cứu định tính \\
\hline 11 & Không mất thời gian gửi xe & Nghiên cứu định tính \\
\hline 12 & Bãi giữ xe & $\begin{array}{l}\text { Jason và Marguerite (2006) và Nghiên } \\
\text { cứu định tính }\end{array}$ \\
\hline 13 & Lựa chọn hàng hoá dễ dàng & $\begin{array}{l}\text { Jason và Marguerite (2006) và Nghiên } \\
\text { cứu định tính }\end{array}$ \\
\hline 14 & Trưng bày hàng hoá hấp dẫn & Koo (2003) \\
\hline 15 & $\begin{array}{l}\text { Giá bán cố định, niêm yết rõ ràng, không phải } \\
\text { trả giá }\end{array}$ & $\begin{array}{c}\text { M. T. Nguyen (2014) và Nghiên cứu định } \\
\text { tính }\end{array}$ \\
\hline 16 & Nhiều sản phẩm mới & $\begin{array}{c}\text { M. T. Nguyen (2014) và Nghiên cứu định } \\
\text { tính }\end{array}$ \\
\hline 17 & Giờ hoạt động (mở cửa, đóng cửa) & $\begin{array}{l}\text { Jason và Marguerite (2006) và Nghiên } \\
\text { cứu định tính }\end{array}$ \\
\hline 18 & Vị trí thuận tiện & $\begin{array}{c}\text { Sinha và Banerjee (2004) và Nghiên cứu } \\
\text { định tính }\end{array}$ \\
\hline 19 & Thời gian mở cửa lâu (24h) & Nghiên cứu định tính \\
\hline 20 & Thái độ phục vụ của nhân viên, người bán & $\begin{array}{l}\text { Jason và Marguerite (2006) và Nghiên } \\
\text { cứu định tính }\end{array}$ \\
\hline 21 & Nhân viên thân thiện, chào hỏi nhiệt tình & Nghiên cứu định tính \\
\hline 22 & Thanh toán nhanh & $\begin{array}{c}\text { Jin và Kim (2003) và Nghiên cứu định } \\
\text { tính }\end{array}$ \\
\hline 23 & Chương trình khuyến mãi & Ugur (2003) và Nghiên cứu định tính \\
\hline
\end{tabular}

Nguồn: Kết quả phân tích dữ liệu điều tra

\subsection{Kết quả nghiên cứu định lự̆ng}

Kết quả thu thập số liệu khảo sát, Kích thước mẫu là 252. Về giới tính, có 130 nam (chiếm $51.6 \%$ mẫu) và 122 nữ (chiếm 48.4\% mẫu). Về thu nhập, có 35 người thu nhập dưới 2 triệu (chiếm 13.9\% mẫu), 69 người thu nhập từ 2 đến dưới 6 triệu (chiếm $27.4 \%$ mẫu); 114 người thu nhập từ 6 đến dưới 10 triệu (chiếm $45.2 \%$ mẫu) và 34 người thu nhập từ 10 triệu trở lên (chiếm 13.5\% mẫu). Về độ tuổi, có 93 người có độ tuổi nhỏ hơn 30 (chiếm $36.9 \%$ mẫu); 81 người có độ tuổi từ 30 đến dưới 40 (chiếm $32.1 \%$ mẫu); 66 người có độ tuổi từ 40 đến dưới 50 (chiếm 26.2\% mẫu); 12 người có độ tuổi từ 50 trở lên (chiếm $4.8 \%$ mẫu). 
Trong phân tích EFA lần 1 với 23 biến quan sát, kết quả phân tích cho thấy có 5 nhân tố được trích tại Eigenvalues là 1.211 và tổng phương sai trích là $48.112 \%$ thấp hơn yêu cầu là $50 \%$. Có 6 biến quan sát có trọng số nhỏ hơn 0.4 là 1) Có thể tự phục vụ̣;2) Có dịch vụ thanh toán điện nước, internet, ATM; 3) Bãi giữ xe; 4) Nhiều sản phẩm mới; 5) Thanh toán nhanh và 6) Chương trình khuyến mãi. Vì vậy các biến quan sát này bị loại. Sau khi loại 6 biến quan sát, kết quả chạy EFA lần 2 cho thấy kết quả có 5 nhân tố được trích tại Eigenvalues là 1.069 và tổng phương sai trích là $57,400 \%$ đạt yêu cầu lớn hơn $50 \%$. Tuy nhiên có 1 biến quan sát nhỏ hơn 0.4 là Có khu vực ăn uống. Nên biến này bị loại.

Sau khi loại biến quan sát Có khu vực ăn uống. Kết quả phân tích EFA lần 3 có 5 nhân tố được trích tại Eigenvalues là 1.056 và tổng phương sai trích là 58,560 \% đạt yêu cầu do lớn hơn $50 \%$. Và không có biến quan sát nào có trọng số nhỏ hơn 0.4 và Cronbach's Alpha của các nhân tố > 0.6 đạt yêu cầu (Hair, Black, Babin, \& Anderson, 2009).

\section{Bảng 2}

Kết quả phân tích EFA

\begin{tabular}{|c|c|c|c|c|c|c|}
\hline \multirow{2}{*}{ STT } & \multirow{2}{*}{ Biến quan sát } & \multicolumn{5}{|c|}{ Factor } \\
\hline & & 1 & 2 & 3 & 4 & 5 \\
\hline 1 & Nơi làm việc, học bài & 0.9 & & & & \\
\hline 2 & Nơi có thể nói chuyện với người quen & 0.773 & & & & \\
\hline 3 & Nơi nghỉ chân thư giãn & 0.761 & & & & \\
\hline 4 & Nơi để gặp gỡ mọi người & 0.691 & & & & \\
\hline 5 & Nơi để giết thời gian & 0.654 & & & & \\
\hline 6 & Có wifi miễn phí, sạc điện thoại & 0.447 & & & & \\
\hline 7 & Không tốn tiền gửi xe & & 0.998 & & & \\
\hline 8 & Không mất thời gian gửi xe & & 0.795 & & & \\
\hline 9 & Lựa chọn hàng hoá dễ dàng & & & 0.915 & & \\
\hline 10 & Trưng bày hàng hoá (hấp dẫn, dễ tìm kiếm) & & & 0.682 & & \\
\hline 11 & $\begin{array}{l}\text { Giá bán cố định, niêm yết rõ ràng, không } \\
\text { phải trả giá }\end{array}$ & & & 0.598 & & \\
\hline 12 & Giờ hoạt động (mở cửa, đóng cửa) & & & & 0.791 & \\
\hline 13 & Vị trí thuận tiện & & & & 0.702 & \\
\hline 14 & Thời gian mở cửa lâu (24h) & & & & 0.642 & \\
\hline 15 & Thái độ phục vụ của nhân viên, người bán & & & & & 0.919 \\
\hline 16 & Nhân viên thân thiện, chào hỏi nhiệt tình & & & & & 0.413 \\
\hline & Eigenvalue & 5.170 & 2.262 & 1.481 & 1.242 & 1.056 \\
\hline & Phương sai trích & 32.315 & 14.138 & 9.254 & 7.762 & 6.603 \\
\hline & Cronbach alpha & $\mathbf{0 . 8 6 3}$ & 0.871 & 0.779 & 0.743 & 0.647 \\
\hline
\end{tabular}

Nguồn: Kết quả xử lý từ dữ liệu điều tra

Tiếp theo, các nhân tố trích được sẽ được đặt tên dựa trên giá trị nội dung của các biến trong và các nghiên cứu lý thuyết được thực hiện trước đó nó (Hair et al., 2009). Tên các nhân tố được đặt tên như sau: nhân tố 1 được đặt tên là Tiện ích, nhân tố 2 được đặt tên là Tiện lợi 
giữ xe, nhân tố 3 được đặt tên là Tiện lợi lựa chọn hàng hoá, nhân tố 4 được đặt tên là Tiện lợi về vị trí và thời gian và nhân tố 5 được đặt tên là Nhân viên thân thiện/Dịch vụ khách hàng. Phương pháp phân tích CFA được sử dụng để tiếp tục phân tích kết quả này.

\section{Bảng 3}

Bảng tóm tắt kết quả kiểm định thang đo

\begin{tabular}{|c|c|c|c|}
\hline Khái niệm & $\begin{array}{c}\text { Số biến } \\
\text { quan sát }\end{array}$ & $\begin{array}{c}\text { Độ tin cậy tổng } \\
\text { hợp }\end{array}$ & Phương sai trích (\%) \\
\hline Tiện ích & 6 & 0.863928418 & 0.518232 \\
\hline Tiện lợi lựa chọn hàng hoá & 3 & 0.787670394 & 0.555159 \\
\hline Tiện lợi giữ xe & 2 & 0.878453985 & 0.783995 \\
\hline Nhân viên & 2 & 0.667035548 & 0.507313 \\
\hline Tiện lợi về vị trí và thời gian & 3 & 0.749834091 & 0.500652 \\
\hline
\end{tabular}

Nguồn: Kết quả xử lý từ dữ liệu điều tra

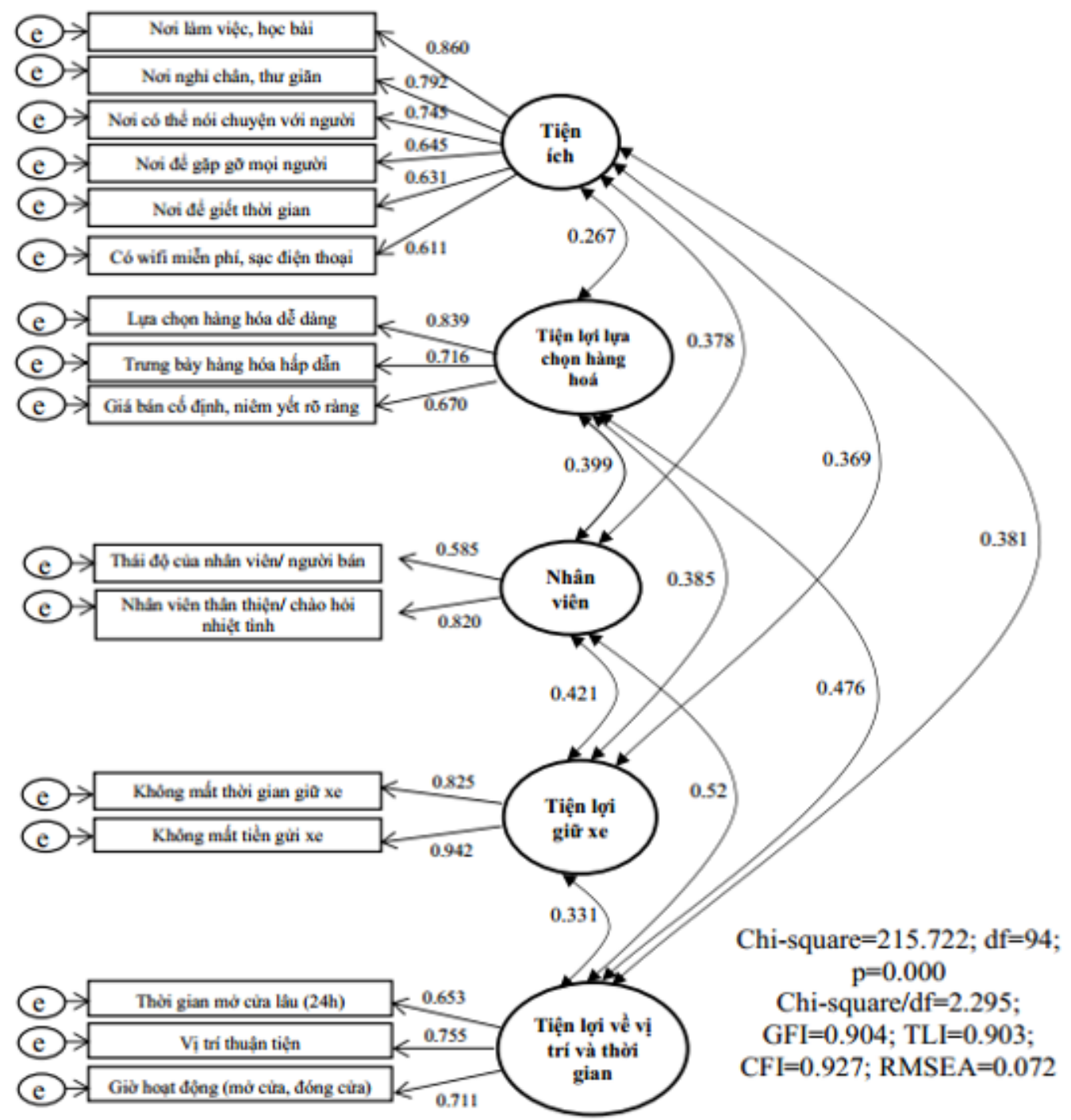

Hình 1. Kết quả phân tích CFA 


\section{Bảng 4}

Mối quan hệ giữa các khái niệm nghiên cứu

\begin{tabular}{|c|c|c|c|c|c|c|}
\hline Nhân viên & <--> & $\begin{array}{c}\text { Tiện lợi lựa } \\
\text { chọn hàng hoá }\end{array}$ & 0.399 & 0.057993069 & 10.36330746 & 0.000000000 \\
\hline Nhân viên & $<-->$ & Tiện ích & 0.378 & 0.058553087 & 10.62283872 & 0.000000000 \\
\hline Nhân viên & <--> & $\begin{array}{c}\text { Tiện lợi về vị trí } \\
\text { và thời gian }\end{array}$ & 0.52 & 0.054022218 & 8.885233166 & 0.000000000 \\
\hline Nhân viên & $<-->$ & Tiện lợi giữ xe & 0.421 & 0.057367552 & 10.09281348 & 0.000000000 \\
\hline $\begin{array}{c}\text { Tiện lợi lựa } \\
\text { chọn hàng } \\
\text { hoá }\end{array}$ & $<-->$ & Tiện ích & 0.267 & 0.06094952 & 12.02634572 & 0.000000000 \\
\hline $\begin{array}{c}\text { Tiện lợi lựa } \\
\text { chọn hàng } \\
\text { hoá }\end{array}$ & $<-->$ & $\begin{array}{c}\text { Tiện lợi về vị trí } \\
\text { và thời gian }\end{array}$ & 0.476 & 0.055621003 & 9.420901631 & 0.000000000 \\
\hline $\begin{array}{c}\text { Tiện lợi lựa } \\
\text { chọn hàng } \\
\text { hoá }\end{array}$ & $<-->$ & $\begin{array}{c}\text { Tiện lợi giữ xe } \\
\text { Tiện ích }\end{array}$ & 0.385 & 0.058370369 & 10.53616772 & 0.000000000 \\
\hline Tiện ích & $<-->$ & $\begin{array}{c}\text { Tiện lợi về vị trí } \\
\text { và thời gian }\end{array}$ & 0.381 & 0.05847526 & 10.58567338 & 0.000000000 \\
\hline $\begin{array}{c}\text { Tiện lợi vợi giữ xe } \\
\text { trí và thời } \\
\text { gian }\end{array}$ & $<-->$ & 0.369 & 0.058782276 & 10.73452817 & 0.000000000 \\
\hline
\end{tabular}

Ghi chú: ML: giá trị ước lượng; se: sai lệch chuẩn; cr: giá trị tới hạn Nguồn: Kết quả xử lý từ dữ liệu điều tra

Mô hình tới hạn các nhân tố và biến quan sát được trích xuất trong phân tích EFA được phân tích. Kết quả phân tích CFA cho thấy mô hình này có giá trị thống kê Chi bình phương là 216,722 với 94 bậc tự do với giá trị $\mathrm{p}=0.000$. Các chỉ số Chi-square/df $=2.295 ; \mathrm{GFI}=0.904$, $\mathrm{TLI}=0.903, \mathrm{CFI}=0.924$ (gần bằng 1 ), RMSEA $=0.072(<0.08)$ nên mô hình được xem là phù hợp, tương thích với dữ liệu thị trường. Các hệ số tương quan và sai lệch chuẩn khác với 1 (xem Bảng 3 và 4 Mối quan hệ giữa các khái niệm nghiên cứu) nên các khái niệm nghiên cứu đạt được giá trị phân biệt. Dựa vào kết quả này có thể thấy các giả thuyết $\mathrm{H1}, \mathrm{H} 2, \mathrm{H} 3, \mathrm{H} 4, \mathrm{H} 5$ đã được hỗ trợ và do đó các thuộc tính bán lẻ của cửa hàng tiện lợi được xác định là Tiện ích; Tiện lợi lựa chọn hàng hóa; Tiện lợi giữ xe; Nhân viên; Tiện lợi về vị trí và thời gian.

Mục tiêu nghiên cứu thứ hai của nghiên cứu này là so sánh sự khác biệt của các thuộc tính bán lẻ của cửa hàng tiện lợi ở thị trường có nền kinh tế chuyển đổi Việt Nam với các thuộc tính bán lẻ của cửa hàng tiện lợi đã được nghiên cứu ở các thị trường các nước phát triển.

Trong nghiên cứu lý thuyết về thuộc tính bán lẻ của cửa hàng tiện lợi ở các nước phát triển như Anh (Wood \& Browne, 2007), Pháp (Gahinet \& Cliquet, 2018), Mỹ (Nielsen, 2017), Canada (Sutton \& Rafaeli, 1988) thì các thuộc tính quan trọng của cửa hàng tiện lợi là vị trí, tiện lợi về thời gian, tốc độ, trải nghiệm, cá nhân hóa và dịch vụ khách hàng. Trong nghiên cứu này, kết quả nghiên cứu cho thấy thuộc tính bán lẻ của cửa hàng tiện lợi ở Việt Nam như vị trí, 
tiện lợi về thời gian, dịch vụ khách hàng là quan trọng tương tự với các nước trên, nhưng các thuộc tính bán lẻ như tốc độ, trải nghiệm và cá nhân hóa chưa được xem là thuộc tính quan trọng ở Việt Nam theo kết quả nghiên cứu định tính và định lượng. Ngoài ra, trong đề tài nghiên cứu này cho thấy các thuộc tính bán lẻ như Tiện ích, Tiện lợi giữ xe, Tiện lợi lựa chọn hàng hóa là quan trọng trong thị trường Việt Nam nhưng chưa được xem có tầm quan trọng ở các nước phát triển, điều này có thể giải thích do văn hóa khác nhau nên cảm nhận về tầm quan trọng của thuộc tính bán lẻ của người tiêu dùng là khác nhau (Jin \& Kim, 2003).

Đối với các nước phát triển ở châu Á như Nhật Bản và Đài Loan, các nghiên cứu trước đây như Hsu và Huang (2006) và Rapp và Islam (2006) cho thấy thuộc tính bán lẻ quan trọng của cửa hàng tiện lợi ở Nhật Bản và Đài Loan là các dịch vụ ngân hàng bán lẻ, dịch vụ thanh toán, dịch vụ đại lý, dịch vụ logistic... tuy nhiên, các thuộc tính này thì chưa được xem là quan trọng và phổ biến trong thị trường Việt Nam và các thuộc tính của cửa hàng tiện lợi ở Việt Nam như Tiện ích, Tiện lợi giữ xe chưa được đánh giá là quan trọng ở Nhật Bản và Đài Loan.

Ngoài ra, nghiên cứu ở một thị trường mới nổi ở Chi Lê (Bianchi, 2009) đề nghị các thuộc tính bán lẻ quan trọng của cửa hàng tiện lợi là Vị trí, nhiều hình thức thanh toán, đậu xe, nhân viên, giờ hoạt động, sạch sẽ và gọn gàng... Nghiên cứu ở Việt Nam cho thấy có sự khác biệt với nghiên cứu ở Chi Lê là thuộc tính bán lẻ như các tiện ích là nơi để gặp gỡ người quen, nơi làm việc thì chưa được xem là quan trọng.

Điểm giống nhau nhất của các nghiên cứu trước đây là hầu như các nghiên cứu này đều đề nghị thuộc tính bán lẻ vị trí là quan trọng (Bianchi, 2009; Deka, 2018; Hsu \& Huang, 2006; Zairis \& Evangelos, 2014). Nghiên cứu này cũng xác nhận vị trí là một thuộc tính quan trọng của cửa hàng tiện lợi ở Việt Nam. Nhưng đối với các thuộc tính khác thì có sự khác biệt trong đánh giá tầm quan trọng như giải thích phần trên.

\section{Kết luận và khuyến nghị}

\subsection{Kết luận}

Nghiên cứu này đã thực hiện được hai mục tiêu đã đề ra là: Đối với mục tiêu thứ nhất, nghiên cứu đã xác định được 05 thuộc tính của cửa hàng tiện lợi tại Việt Nam: đó là Tiện ích, Tiện lợi giữ xe, Tiện lợi lựa chọn hàng hóa, Tiện lợi về vị trí và thời gian và Dịch vụ khách hàng. Đối mới mục tiêu thứ hai, nghiên cứu cũng đã so sánh được sự giống nhau và khác nhau của các thuộc tính bán lẻ cửa hàng tiện lợi ở thị trường có nền kinh tế chuyển đổi Việt Nam với các thuộc tính bán lẻ của cửa hàng tiện lợi đã được nghiên cứu ở các thị trường các nước phát triển.

\subsection{Khuyến nghị}

\subsubsection{Khuyến nghị cho nhà bán lẻ và nhà quản lý}

Các thuộc tính của cửa hàng tiện lợi tìm được trong nghiên cứu này giúp cho các doanh nghiệp bán lẻ cửa hàng tiện lợi nhận biết được các thuộc tính bán lẻ quan trọng của hình thức bán lẻ cửa hàng tiện lợi đối với người tiêu dùng. Các doanh nghiệp bán lẻ có thể tập trung vào các thuộc tính này để thu hút, làm thoả mãn người tiêu dùng và tăng lợi thế cạnh tranh. Cụ thể như sau: 
Kết quả nghiên cứu cho thấy, vị trí và thời gian là một thuộc tính rất quan trọng đối với người tiêu dùng khi chọn cửa hàng tiện lợi. Các nhà bán lẻ nên mở rộng hệ thống nhiều cửa hàng để có thể làm cho khách hàng tiếp cận dễ dàng cửa hàng tiện lợi với vị trí thuận tiện, gần nhà, nơi làm việc, trường học... Vinmart+ đang được xem là dẫn đầu về số lượng cửa hàng tiện lợi ở Việt Nam khi số lượng cửa hàng đạt được hơn 800, vượt trội hơn nhiều đối thủ khác như Circle K và Shop\&Go (Viet Hung, 2018).

Thuộc tính Tiện ích được tìm ra trong nghiên cứu này được xem là rất quan trọng. Nhiều khách hàng chọn cửa hàng tiện lợi để mua sắm vì có chỗ ngồi thuận tiện, wifi miễn phí (Viet Hung, 2018). Theo kết quả nghiên cứu định tính, nhiều hệ thống cửa hàng tiện lợi không cung cấp tốt được các tiện ích này cho người mua sắm nên không được đánh giá cao. Các hệ thống cửa hàng tiện lợi nên tạo ra nhiều tiện ích hơn cho người mua sắm để đáp ứng tốt được nhu cầu của khách hàng.

Tiện lọi giữ xe, thuộc tính này được đánh giá có tầm quan trọng cao của người mua sắm, tuy nhiên nhiều hệ thống cửa hàng tiện lợi có diện tích nhỏ nên không có nhiều không gian để cho khách hàng đậu xe. Các cửa hàng tiện lợi nên có thêm các khu vực để giúp người mua sắm dễ dàng đậu xe hơn.

Tiện lọii lựa chọn hàng hóa, mặc dù thuộc tính Tiện lợi lựa chọn hàng hóa được đánh giá có tầm quan trọng cao của người tiêu dùng khi mua sắm ở cửa hàng tiện lợi theo kết quả nghiên cứu định tính, tuy nhiên vẫn còn nhiều hệ thống cửa hàng tiện lợi việc lựa chọn hàng vẫn chưa thật sự dễ dàng. Người tiêu dùng vẫn còn phải hỏi nhân viên về mặt hàng cần mua được đặt ở đâu. Nên các hệ thống cửa hàng tiện lợi cần sắp xếp, trưng bày hàng hóa tốt hơn để dễ dàng cho người tiêu dùng tìm kiếm.

Nhân viên thân thiện/Dịch vụ khách hàng là một thuộc tính quan trọng. Theo nghiên cứu định tính, vẫn có sự khác nhau về đánh giá chất lượng dịch vụ khách hàng giữa các hệ thống. Do vậy, các hệ thống cửa hàng tiện lợi nên có các chương trình huấn luyện, đào tạo để nâng cao chất lượng dịch vụ khách hàng.

Đối với các nhà quản lý, việc hiểu biết các thuộc tính bán lẻ của cửa hàng tiện lợi giúp cho việc lập kế hoạch và phát triển thị trường bán lẻ hiệu quả hơn. Mỗi hình thức bán lẻ có những lợi thế riêng, thoả mãn các nhu cầu tiêu dùng riêng. Việc phát triển hình thức bán lẻ tiện lợi sẽ góp phần tạo ra nhiều lựa chọn và thỏa mãn tốt hơn nhu cầu mua sắm của người tiêu dùng.

Để phát triển hệ thống cửa hàng tiện lợi, nhà nước cần có các chính sách ưu đãi về thuế, thủ tục, tạo điều kiện cho các nhà bán lẻ dễ dàng hơn thuê mặt bằng, mở rộng hệ thống cửa hàng. Giúp các cửa hàng tiện lợi có được vị trí tiện lợi trong việc cung cấp dịch vụ cho người tiêu dùng. Việc phát triển hệ thống bán lẻ cửa hàng tiện lợi nói riêng và các hệ thống bán lẻ nói chung mang lại nhiều lợi ích như phát triển các ngành khác như logistic, vận chuyển, sản xuất, thương mại và tạo cơ hội việc làm cho hàng triệu lao động trong nước.

Ngoài ra nhà nước cần phát triển cơ sở hạ tầng giao thông, phương tiện vận chuyển, chuyên chở, cung cấp các dịch vụ thanh toán tài chính tiện lợi hơn người tiêu dùng để họ có thể dễ dàng hơn trong việc đi mua sắm. 
Trong kết quả nghiên cứu, các thuộc tính bán lẻ quan trọng như vị trí, tiện ích...đòi hỏi các hệ thống cửa hàng tiện lợi phải có một nguồn lực tài chính mạnh để có thể thực hiện. Các nhà quản lý cần có các chính sách ưu đãi về tín dụng để giúp các hệ thống cửa hàng tiện lợi nhỏ phát triển và cạnh tranh công bằng.

\subsubsection{Hạn chế và hướng nghiên cưu tiếp theo}

Nghiên cứu này nghiên cứu các thuộc tính bán lẻ quan trọng của hình thức bán lẻ cửa hàng tiện lợi ở Việt Nam sử dụng mẫu được thu thập ở Thành phố Hồ Chí Minh. Các nghiên cứu tiếp theo sẽ được thực hiện ở các thành phố khác như Hà Nội, Đà Nẵng... để kiểm định tính tổng quát của mô hình nghiên cứu. Hình thức chọn mẫu trong nghiên cứu này là thuận tiện nên còn hạn chế trong việc thu thập số liệu mang tính đại diện cao cho thị trường. Nghiên cứu tiếp theo sẽ chọn mẫu theo xác suất để kiểm định tính đại diện của mô hình nghiên cứu. Do nghiên cứu có hạn chế về tài chính và thời gian, nên mẫu được lựa chọn chưa được lớn. Nghiên cứu tiếp theo sẽ tăng kích cỡ của mẫu lớn hơn.

\section{Tài liệu tham khảo}

Akinobu, T. (1998). Development of new store types: The role of convenience stores in Japan. GeoJournal, 45(4), 317-325.

Antonios, Z. G., \& Prontzas, E. (2014). Consumer behaviour toward convenience store chains in Greece. EuroMed Journal of Business, 9(2), 175-197.

Batra, R. (1997). Marketing issues and challenges in transitional economies. Journal of International Marketing, 5(4), 95-114.

Bianchi, C. C. (2009). Investigating consumer expectations of convenience-store attributes in emerging markets: Evidence in Chile. Journal of International Consumer Marketing, 21(4), 309-320.

Cheng, J. M.-S., Blankson, C., Sutikno, B., \& Wang, M. C.-H. (2009). Hybrid convenience stores - The changing role of convenience stores in Taiwan. Asia Pacific Journal of Marketing and Logistics, 21(3), 417-432.

David, D., \& Kirby, A. (1986). Convenience stores: The polarisation of British retailing. Retail and Distribution Management, 14(2), 7-12.

Deka, P. K. (2018). Factors influencing consumers' choice of retail store format in Assam, India. IUP Journal of Marketing Management, 17(2), 22-37.

Gahinet, M.-C., \& Cliquet, G. (2018). Proximity and time in convenience store patronage: Kaïros more than chronos. Journal of Retailing and Consumer Services, 43(1), 1-9.

Garves, S. M. (2017). Convenience stores: A landscape perspective. Yearbook of the Association of Pacific Coast Geographers, 79(1), 134-152. 
GSO. (2017). Thông cáo báo chí tình hình kinh tế - xã hội năm 2017. 2018 [Press release on socio-economic situation in 2017. 2018]. Retrieved June 20, 2019, from http://www.gso.gov.vn/default.aspx?tabid=382\&idmid=\&ItemID=18667

Hair, Jr, J. F., Black, W. C., Babin, B. J., \& Anderson, R. E. (2009). Multivariate data analysis. New York, NY: Pearson Publications.

Hsu, Y. C., \& Huang, H. L. (2006). Alternative futures for convenience store management in Asia: An E-business perspective. Journal of Futures Studies, 10(3), 79-88.

Jason, M. C., \& Marguerite, M. (2006). Consumer demographics, store attributes, and retail format choice in the US grocery market. International Journal of Retail \& Distribution Management, 34(6), 434-452.

Jin, B., \& Kim, J. O. (2003). A typology of Korean discount shoppers: Shopping motives, store attributes, and outcomes. International Journal of Service Industry Management, 14(3/4), 396-419.

Kim, Y.-K., \& Kang, J. (1995). Consumer perception of shopping costs and its relationship with retail trends. Journal of Shopping Center Research, 2(1), 27-61.

Koo, D.-M. (2003). Inter-relationships among store images, store satisfaction, and store loyalty among Korea discount retail patrons. Asia Pacific Journal of Marketing and Logistics, $15(4), 42-71$.

NACS. (2016). What is a convenience store? 2018. Retrieved June 23, 2019, from https://www.convenience.org/ Research/What-is-a-Convenience-Store

Nguyen, A. (2015). Siêu thị mini, cửa hàng tiện lợi sẽ lên ngôi [Mini supermarkets and convenience stores will be crowned]. Retrieved June 24, 2019, from http://www.kinhtedothi.vn/sieu-thi-mini-cua-hang-tien-loi-se-len-ngoi-28197.html

Nguyen, M. T. (2014). Shopping motivations, retail attributes, and retail format choice in a transitional market: Evidence from Vietnam (Doctoral dissertation, Western Sydney University, Sydney, Australia). Retrieved from June 25, 2019, from https://researchdirect.westernsydney.edu.au/islandora/object/uws\%3A35473

Nguyen, T. M. T., \& Nguyen, T. D. (2003). Measurement of service quality of supermarkets in Vietnam - A customer perspective. Paper presented at Vietnam National University, Ho Chi Minh.

Nguyen, T. T. M., Nigel, J. B., \& Nguyen, T. D. (2007). Hedonic shopping motivations, supermarket attributes, and shopper loyalty in transitional markets. Asia Pacific Journal of Marketing and Logistics, 19(3), 227-239.

Nielsen. (2017). How U.S. convenience stores can stay ahead of the retail pack. Retrieved May 20, 2019, from https://www.nielsen.com/us/en/insights/news/2017/how-us-conveniencestores-can-stay-ahead-of-the-retail-pack.html/

Ogawa, S. (2002). The hypothesis-testing ordering system: A new competitive weapon of Japanese convenience stores in a New Digital Era. Industrial Relations, 41(4), 579-674. 
Rapp, W. V., \& Islam, M. (2006). Japanese mini-banks: Retail banking services through convenience stores. Asian Business \& Management, 5(2), 187-206.

Reimers, V. (2014). A consumer definition of store convenience (finally). International Journal of Retail \& Distribution Management, 42(4), 315-333.

Seiders, K., Berry, L. L., \& Gresham, L. G. (2000). Attention, retailers! How convenient is your convenience strategy? Sloan Management Review, 43(3), 79-89.

Sinha, P. K., \& Banerjee, A. (2004). Store choice behaviour in an evolving market. International Journal of Retail \& Distribution Management, 32(10), 482-494.

Sutton, R. I., \& Rafaeli, A. (1988). Untangling the relationship between displayed emotions and organizational sales: The case of convenience stores. Academy of Management Journal, 31(3), 461-487.

Ugur, Y. (2003). A multi-attribute approach to understanding shopper segments. International Journal of Retail \& Distribution Management, 31(11/12), 541-548.

Viet Hung (2018). Ai đang thống trị thị trương của hàng tiện lọi Việt Nam? [Who is dominating the Vietnamese convenience store market?]. Retrieved June 21, 2019, from https://theleader.vn/ai-dang-thong-tri-thi-truong-cua-hang-tien-loi-viet-nam1546228046034.htm

Wood, S., \& Browne, S. (2007). Convenience store location planning and forecasting - A practical research agenda. International Journal of Retail \& Distribution Management, $35(4), 233-255$.

Zairis, A. G., \& Evangelos, P. (2014). Consumer behaviour toward convenience store chains in Greece. EuroMed Journal of Business, 9(2), 175-197.

Zielke, S. (2010). How price image dimensions influence shopping intentions for different store formats. European Journal of Marketing, 44(6), 748-770. 